\title{
Dynamics and control in precision mechanics
}

\author{
André Preumont*,*** - Bruno de Marneffe* \\ Gonçalo Rodrigues* - Houssein Nasser** \\ Arnaud Deraemaeker* \\ * ULB - Active Structures Laboratory, Brussels, Belgium \\ ** Centre de Recherche Public Henri Tudor, Luxembourg \\ *** INSA de Lyon, France \\ andre.preumont@ulb.ac.be
}

\begin{abstract}
This paper briefly describes three projects developed at the Active Structures Laboratory of $U L B$, in connection with precision mechanics. The first one considers the integration of piezoelectric fibers in composite structures for space applications; the second consists of an active piezoelectric bimorph mirror for adaptive optics, and the third considers the vibration isolation of sensitive payloads in spacecraft.

RÉSUMÉ. Ce papier décrit brièvement trois projets développés au Laboratoire des Structures Actives de l'ULB, en relation avec la mécanique de précision. Le premier considère l'intégration de fibres piézoélectriques dans des structures composites pour applications spatiales; le second consiste en un miroir actif bimorphe piézoélectrique pour l'optique adaptative et le troisième s'intéresse à l'isolation vibratoire des charges utiles de précision dans les satellites.

KEYWORDS: piezoelectric fibers, piezoelectric shells, mixing rules, bimorph mirror, adaptive optics, Zernike modes, vibration isolation, sky-hook damper, relaxation isolator, Stewart platform.

MOTS-CLÉS: fibres piézoélectriques, coques piézoélectriques, règles des mélanges, miroir bimorphe, optique adaptative, modes de Zernike, microvibration, isolation vibratoire, hexapode.
\end{abstract}

DOI:10.3166/REMN.17.597-611 @ 2008 Lavoisier, Paris 


\section{Introduction}

The availability of smart materials has opened new horizons in the development of active devices, and precision engineering is likely to greatly benefit from them. However, taking full advantage of these new emerging capabilities requires improved modelling techniques for multi-physics phenomena as well as new fabrication techniques. This paper briefly describes three projects currently under way at the Active Structures Laboratory of ULB, in connection with precision mechanics. The first project considers the integration of piezoelectric fibers in composite structures for space applications; it was motivated by the current inability to predict the open-loop zeros in active structures involving piezoelectric shells. New, simple mixing rules have been developed for the calculation of homogenized piezoelectric properties of piezoelectric laminates. The mismatch of dielectric properties between the matrix and the fibers appears to be a significant problem for circular fibers. The second project is part of an effort to use lighter, controllable reflecting surfaces in space telescopes, but also to produce cheaper mirrors for adaptive optics. The test article consists of an active piezoelectric bimorph mirror with honeycomb electrodes. The capability to reproduce given aberrations from numerical data is demonstrated experimentally. The third project considers the vibration isolation of sensitive payloads in spacecraft. It attempts to simplify the control electronics of active isolation platforms by using a passive system which mitigates the resonance peak of the isolator, while keeping a high frequency roll off of $-40 \mathrm{~dB} /$ decade. The concept is based on a electromagnetic realization of a relaxation isolator. The six-axis isolation is achieved with a Stewart platform with cubic architecture.

\section{Modeling of structures with piezocomposite actuators and sensors}

Piezoelectric actuators and sensors have been widely used in active vibration control applications. PZT ceramics are commonly used due to their good actuation capability and very wide bandwidth. The major drawbacks of these ceramics are their brittle nature, and the fact that they cannot be easily attached to curved structures. In order to overcome these drawbacks, two techniques have been developped : (i) screen printing green PZT before sintering which requires that the part be heated at $900{ }^{\circ} \mathrm{C}$ for sintering, and (ii) using PZT fibers in conformal piezocomposites which can be glued on curved structures.

\subsection{Piezofiber composite actuators and sensors}

The first piezocomposite used the $d_{31}$ mode of the piezoelectric fibers (Figure 1(a)). The electric field is applied in the direction perpendicular to the plane of the actuator, while the induced strain is in the plane. A major problem with this kind of configuration comes from the very large difference in the dielectric permittivities of the piezoelectric fiber and the epoxy matrix. This results in a drastic reduction of the 
(a)

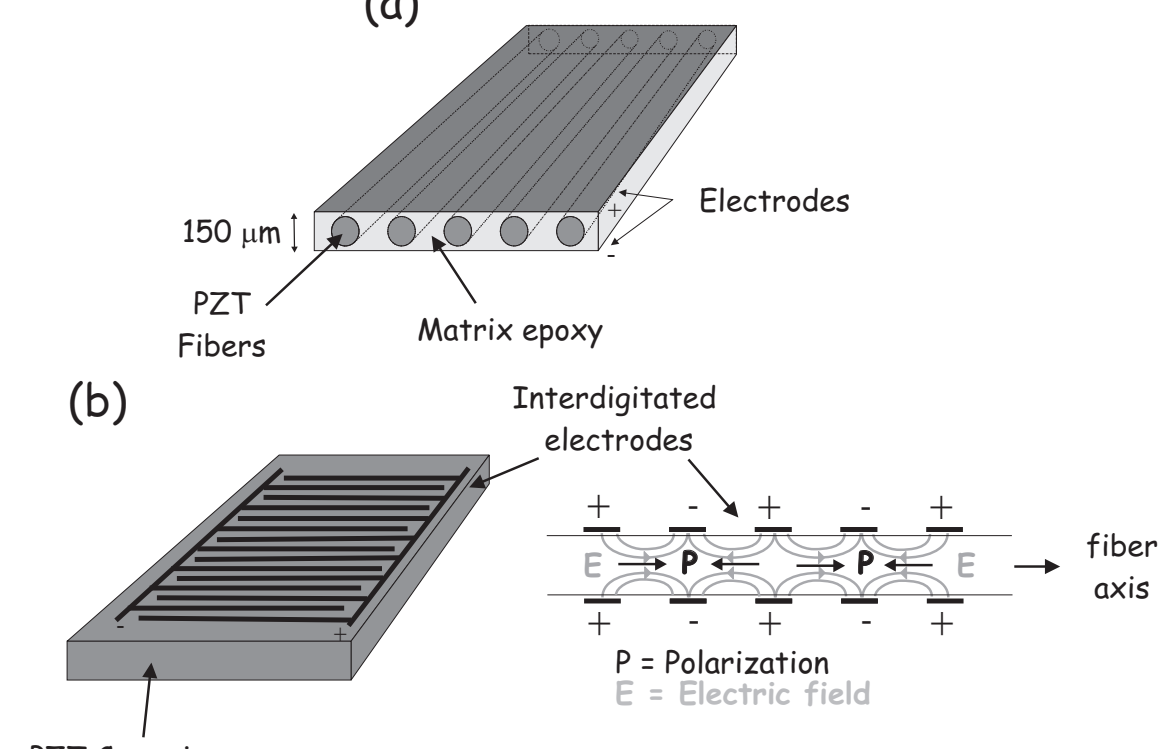

PZT Ceramic

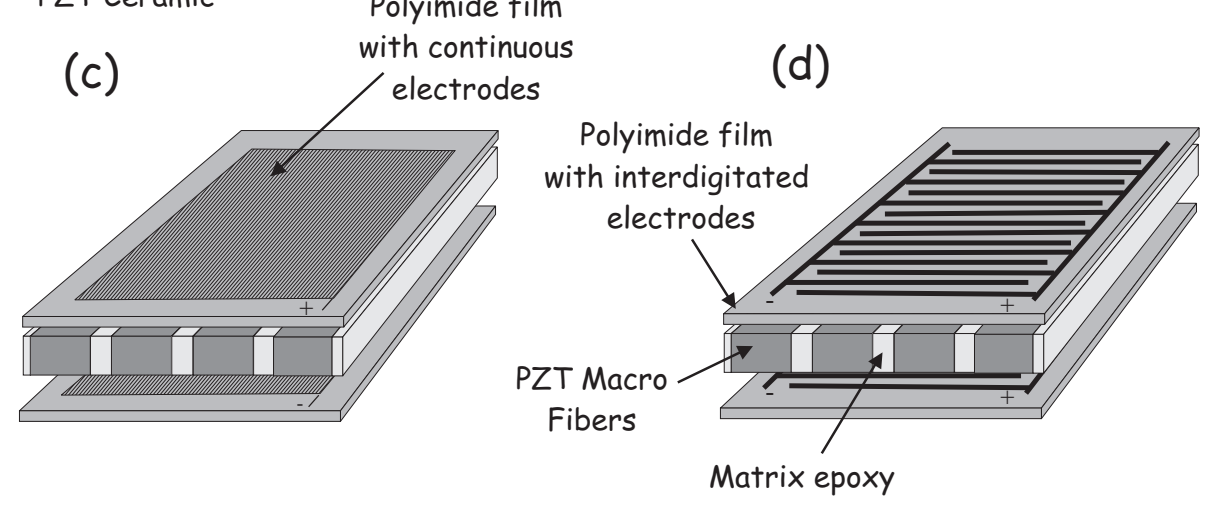

Polyimide film

Figure 1. a) Piezofiber composite using the $d_{31}$ mode, b)Interdigitated electrodes concept and direction of the electric field lines, Layout of Macro Fiber Composites transducers : (c) $d_{31} M F C,(d) d_{33} M F C$

electric field applied to the active fiber, even for a very small layer of epoxy trapped between the electrodes and the fibers (Figure 2: a $1 \mu \mathrm{m}$ thick layer of epoxy results in a loss of $90 \%$ of the electric field in the fiber).

A new principle was proposed in (Bent et al., 1997) in which the fibers are used in the $d_{33}$ mode. The electric field is applied in the direction of the fibers (in the plane of the actuator) through so-called interdigitated electrodes (Figure 1(b)). The actuator 
(a)

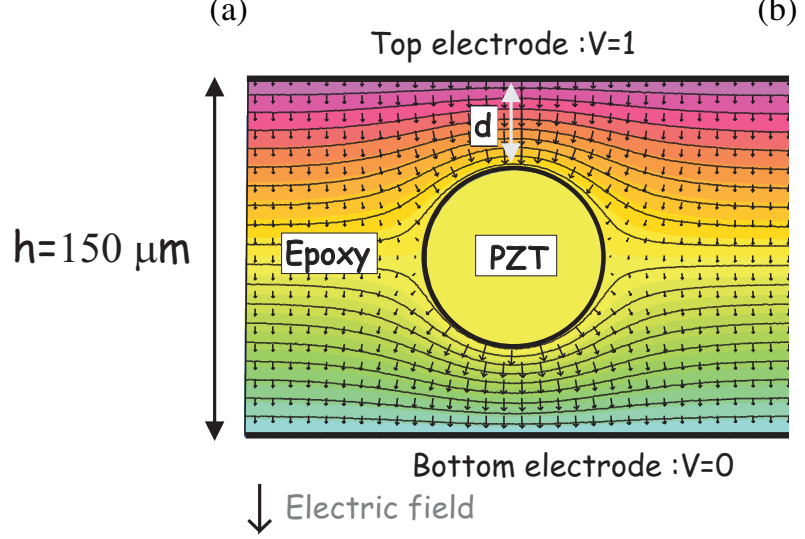

(b)

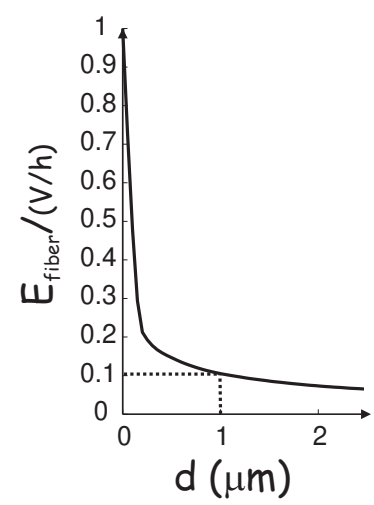

Figure 2. Effect of a small layer of epoxy trapped between the electrode and the piezofiber. (a) Equipotential surfaces in piezocomposite, (b) Reduction of the effective electric field with the thickness $d$ of matrix material between the electrode and the fiber

performance is enhanced due to the high value of the $d_{33}$ coefficient which is usually 2 to 3 times higher than the $d_{31}$. On the other hand, high voltages need to be used, because of the spacing of the fingers of the electrodes which is 5 to 6 times higher than the thickness of the transducer.

Even with this new design, the efficiency is still reduced due to the dielectric mismatch. Different solutions have been studied in order to improve the performance of the actuator, but in all cases, the manufacturing process was complicated and not repeatable.

In parallel to these investigations, the so-called MFCs (Macro Fiber Composites) have been developped (Wilkie et al., 2000) (Figure 1(c-d)). The main difference with the previous attempts is that the fibers are rectangular, and diced from a regular ceramic. The manufacturing process is quite cheap and repeatable, and the shape of the fibers together with the manufacturing process allows to put the fibers in direct contact with the electrodes. Both $d_{31}$ and $d_{33}$ actuators and sensors have been developped and are currently sold by the german company Smart Material (http://www.smartmaterial.com).

\subsection{Properties of Piezocomposite transducers}

The analysis of multi-layer shells including piezoelectric layers can be performed in commercial finite element softwares (Piefort, 2001) such as SAMCEF 
(http://www.samcef.com) or Matlab-SDT (http://www.sdtools.com). This requires to have access to homogenized mechanical as well as electrical properties of the piezocomposite transducers which are not available in the datasheet. In order to overcome this problem, we have developped very simple mixing rules for the prediction of homogenized in plane properties of $d_{31}$ MFCs (Deraemaeker et al., 2007).

\subsection{Example : Modelling of a composite boom equipped with piezocomposite actuators and sensors}

(a)

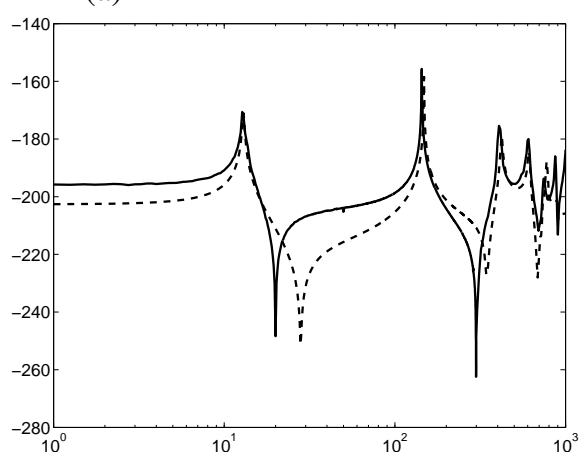

(b)

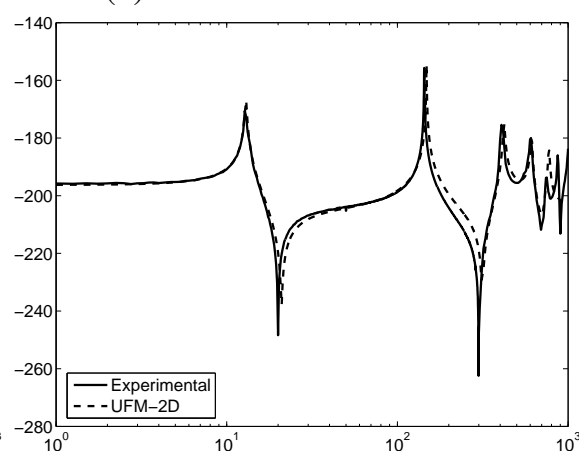

Figure 3. Comparison between the experimental and the numerical transfer function between two opposite patches : (a) using datasheet information, (b) using homogenized properties

The structure is a cylindrical composite boom equipped with $d_{31}$-MFC actuators and sensors described in more details in (Deraemaeker et al., 2007). In order to design the controller and assess its performances, accurate modeling of the transfer function between the actuators and sensors is necessary. Some properties of the MFC patches are not available in the datasheet. However, they turn out to have a significant impact on the modelling of the test structure (Figure 3(a)). In particular, the modeling of the open-loop zeros is poor. The mechanical and electrical properties of $d_{31}$ MFCs have been computed using the newly developped mixing rules, and introduced in a finite element model of the boom using shell composite piezoelectric elements in SAMCEF. Figure 3(b) shows the very good match between the predicted and measured transfer function between two MFCs. In particular, the poles and zeros are well predicted, which is essential in control applications.

\subsection{Future developments}

The main problem with the current design of piezocomposite actuation is due to the dielectric mismatch between the fiber and the matrix which makes the manufacturing process of such devices quite complicated. Improvements could be achieved 
by looking in two directions : new materials (alternative active materials are currently under development), and new material architectures allowing to reduce the effect of dielectric mismatch.

\section{Adaptive Optics}

Adaptive optics employs deformable mirrors to compensate the aberration of astronomical images caused by the turbulence of the earth atmosphere. The requirements in terms of the accuracy of the produced shapes can reach the order of the dozens of nanometers (Roddier, 1999).

The technology of most deformable mirrors is based on two main actuation principles (Figure 4). The monolithic reflecting surface pushed and pulled by linear piezoelectric actuators has proved to have the highest performance in terms of complexity of shapes generated and the temporal rate of correction. However, the complexity of the linear piezoelectric actuators makes it expensive and not very reliable. On the other hand, bimorph mirrors are formed by a piezoelectric layer which is solidary with a passive reflecting substrate. Their simplicity makes them easier and less costly to produce and more robust. The in-plane deformation of the piezoelectric layer with an off-set with respect to the neutral plane results in a bending moment applied along the contour of the electrode where the constant voltage is applied. The bending moment is proportional to the intensity of the locally applied voltage.

The tailoring of the electrodes deposited on the back of the active layer plays a key role on the types of shapes that can be generated. Layouts of actuators with the shapes of a keystone as depicted in figure 5 are specially suited to the generation of the optical aberrations like those described by the polynomials of Zernike and are used in the majority of the existing bimorph mirrors. On the other hand, a layout with the shape of a honeycomb provides a homogeneous geometry which may be extended to larger, segmented optics, and thus is an excellent test bench for experiments on decentralized shape control.

The application of the finite element method for modelling the response of piezoelectric laminate shells has already been addressed in the previous section; in the

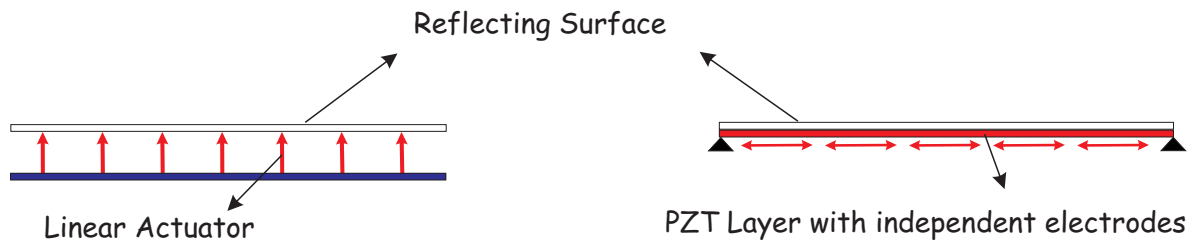

Figure 4. Main principles of actuation used in deformable mirrors. Left: piston-type actuation. Right: in-plane bimorph actuation 


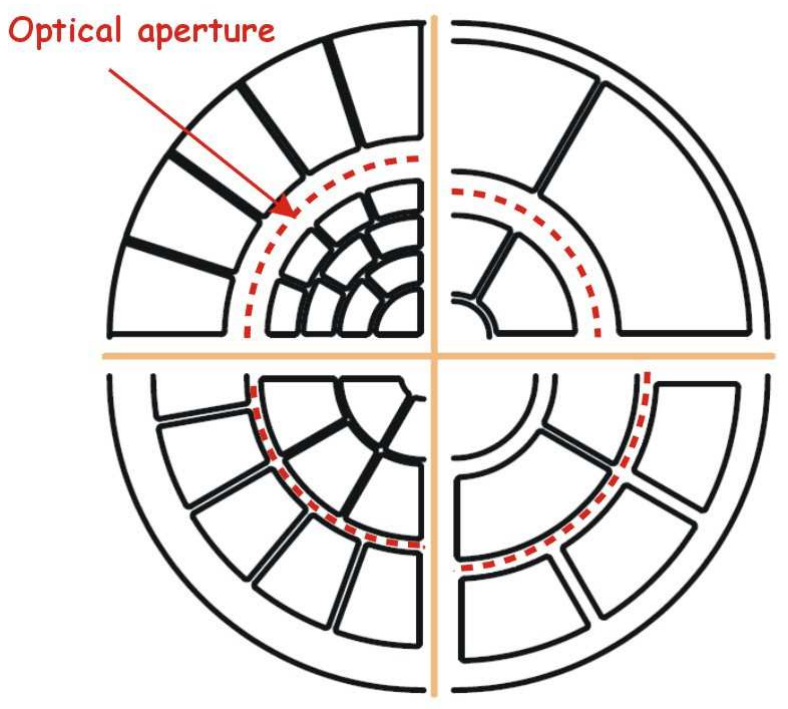

Figure 5. Electrode layouts used in commercial adaptive optics using a bimorph designs. Each quarter shows a different keystone design with circular symmetry

present case, the PZT layer is homogeneous and isotropic. This allows to construct numerically the Jacobian of the adaptive mirror that is the influence matrix relating the voltage applied to individual electrodes and the optical aberrations known as the Zernike modes of the surface. This Jacobian is used in the closed-loop correction of the optical aberrations.

\subsection{Quasi-static wavefront control}

Once the Jacobian matrix relating the optical aberrations $z_{i}$ (Zernike modes) to the electrode voltages $v_{j}$ is available,

$$
z_{i}=J_{i j} v_{j}
$$

the quasi-static correction is readily achieved with the pseudo-inverse:

$$
v_{j}=J_{j i}^{+} z_{i}
$$

provided that the optical aberrations $z_{i}$ are available. These are obtained from a wavefront sensor the most popular of which is the Shack-Hartmann. 


\subsection{Bimorph silicon mirror}

The numerical results shown herein are representative of the experimental demonstrator of adaptive optics under development in our laboratory. Figure 6 shows the 91 honeycomb gold electrodes at the back of the mirror, prior to soldering the control wires. The bimorph mirror itself was manufactured by the Division of Ceramic Technology of the Fraunhofer Institute IKTS in Dresden, and results from the deposition of the gold electrodes and piezoelectric patches on a silicon wafer by screen-printing. The figure also indicates the relative position between the limits of the wafer, the simple support provided by two rubber O-rings, the active optical aperture and the actuator layout.

Figure 7 shows the optical aberrations of astigmatism and trefoil. The simulations obtained by finite elements are presented on the left and include the deformation field in the region surrounding the optical aperture. The open-loop experimental results of deformations within the optical aperture are presented on the right and demonstrate the capability of this mirror for generating these aberrations accurately from numerical

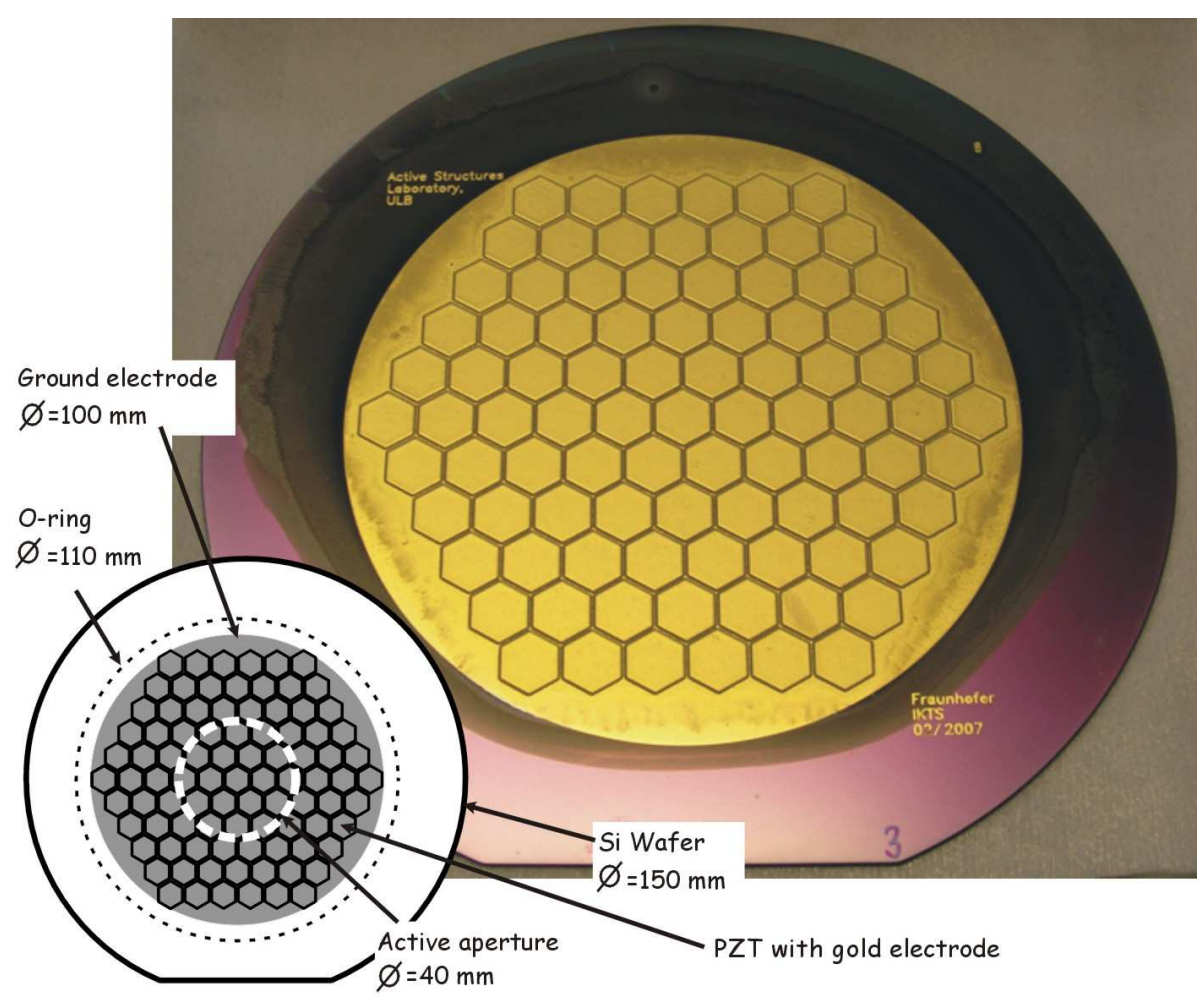

Figure 6. Honeycomb actuator layout of the of ASL bimorph mirror 
data alone. The experimental results were obtained by a phase shift Schlieren (NIMO, from Lambda-X).
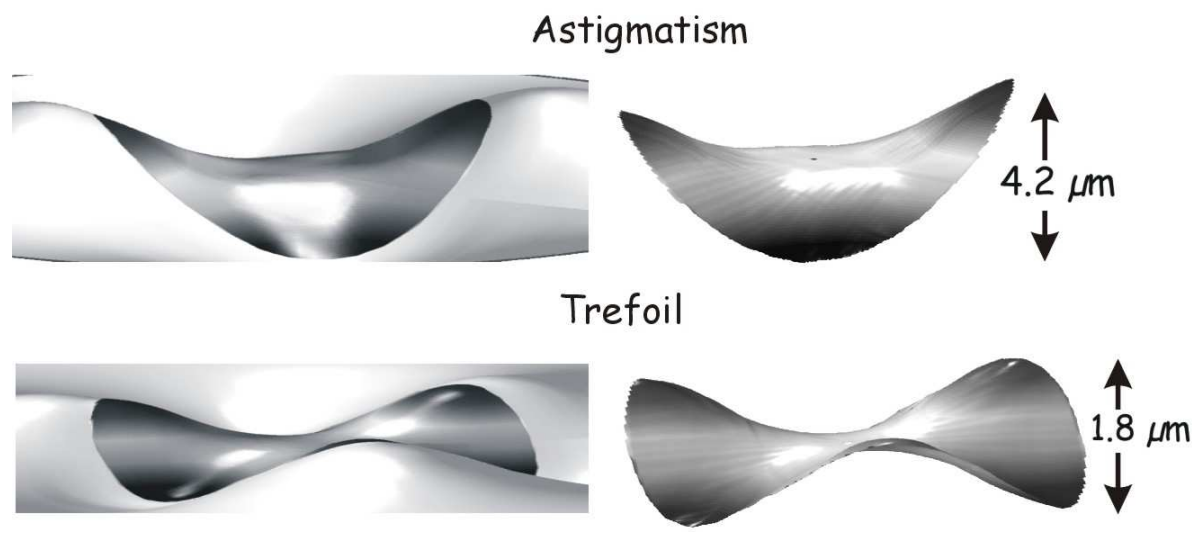

Figure 7. Generation of the optical aberrations of astigmatism and trefoil. Left: finite element simulations (SAMCEF). Right: experimental results (NIMO)

\section{Vibration Isolation}

\subsection{Background}

The micro-vibrations generated by the rotating wheels of the attitude control system have been identified as a major source of disturbances affecting the performance of precision payloads on spacecrafts. Vibration isolation is a classical problem which has received a lot of attention over the years, however, space applications have special requirements in terms of environment, reliability, etc. This project aims at achieving performance as close as possible of an active system with a passive one which does not require electronics, and whose architecture minimizes its sensitivity to space environment, particularly thermal gradients.

The simplest passive single-axis isolator is that of Figure 8(a); it consists of a spring in parallel with a viscous damper. The disturbance is the displacement $X_{2}$ of the supporting structure $m$, and the system output is the displacement $X_{1}$ of the sensitive equipment $M$. The Transmissibility $X_{1} / X_{2}$ of this system is, in Laplace variables:

$$
\frac{X_{1}}{X_{2}}=\frac{c s+k}{m s^{2}+c s+k}=\frac{1+2 \xi s / \omega_{n}}{1+2 \xi s / \omega_{n}+s^{2} / \omega_{n}^{2}}
$$

with the usual notations $\omega_{n}^{2}=k / M$ and $2 \xi \omega_{n}=c / M\left(\omega_{n}\right.$ is the natural frequency of the spring-mass system when $m$ is clamped). The foregoing transmissibility illustrates 


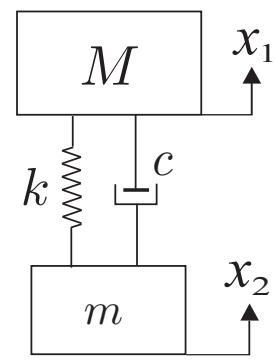

(a)

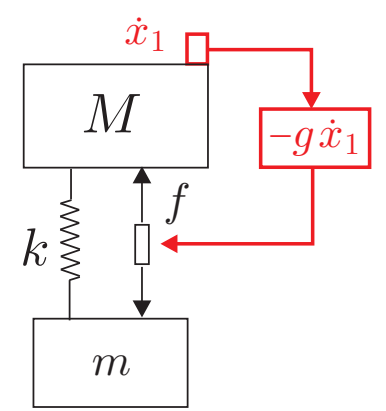

(b)

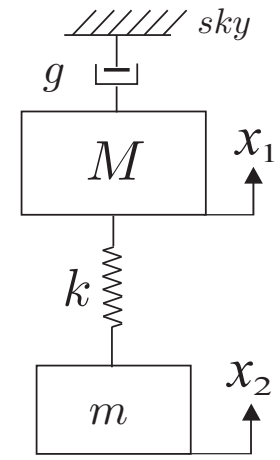

(c)

Figure 8. (a) Single-axis passive isolator. (b) Sky-hook isolator. (c) Equivalent representation of the sky-hook

the design trade-off: the resonance peak at $\omega_{n}$ may be reduced by increasing the passive damping $\xi$, but this is at the expense of reducing the high-frequency attenuation rate from $-40 \mathrm{~dB} / \mathrm{dec}$ to $-20 \mathrm{~dB} / \mathrm{dec}$.

\subsection{Single-axis active isolation}

The behavior of the suspension may be improved actively in the way described at Figure $8(\mathrm{~b})$. The passive damper has been replaced by a force actuator and a feedback loop based on the absolute velocity of the payload (it can be measured with a geophone, or integrated from an accelerometer). This configuration is known as the "sky-hook" damper. The forces acting on the payload side are equivalent to those of Figure 8(c).

This control strategy works well for rigid bodies, but its stability is no longer guaranteed when the structures connected by the isolator are flexible, which is typically the case for large space structures. This situation, however, may be tackled elegantly by changing the velocity feedback by an integral force feedback (IFF) (Preumont, 2002), as illustrated in Figure 9. This control strategy is equivalent to the velocity feedback when the two bodies are rigid, and it has guaranteed stability when the structures connected by the isolator are flexible. The transmissibility of the single-axis isolator based on IFF is given by

$$
\frac{X_{1}(s)}{X_{2}(s)}=\left[\frac{M}{k} s^{2}+\frac{M}{k} g s+1\right]^{-1}
$$

which exhibits an attenuation rate of $-40 \mathrm{~dB} / \mathrm{decade}$ at high frequency, and the overshoot at resonance may be adjusted by the control gain $g$. 


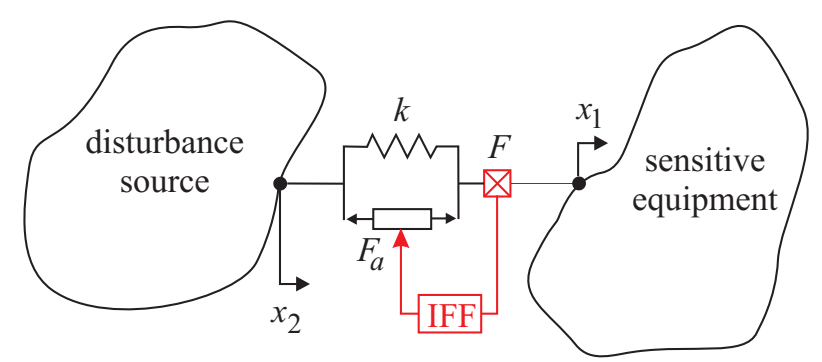

Figure 9. Two arbitrary flexible structures connected by a single-axis soft isolator with force feedback.

\subsection{Six-axis isolation}

The IFF control strategy may be applied to several d.o.f. in a decentralized manner. An elegant way to do this is to use a Gough-Stewart platform with each leg being a single-axis isolator, Figure 10, with decentralized IFF control with the same gain for all loops. This architecture has been manufactured and tested in parabolic flight on behalf of ESA (Preumont et al., 2007). A typical transmissibility plot (in the vertical direction) is shown in Figure 11, which compares experimental results with numerical simulations; note that the latter require an accurate F.E. model up to the first transversal mode at the minimum, that is $500 \mathrm{~Hz}$ in this case. This isolator pro-

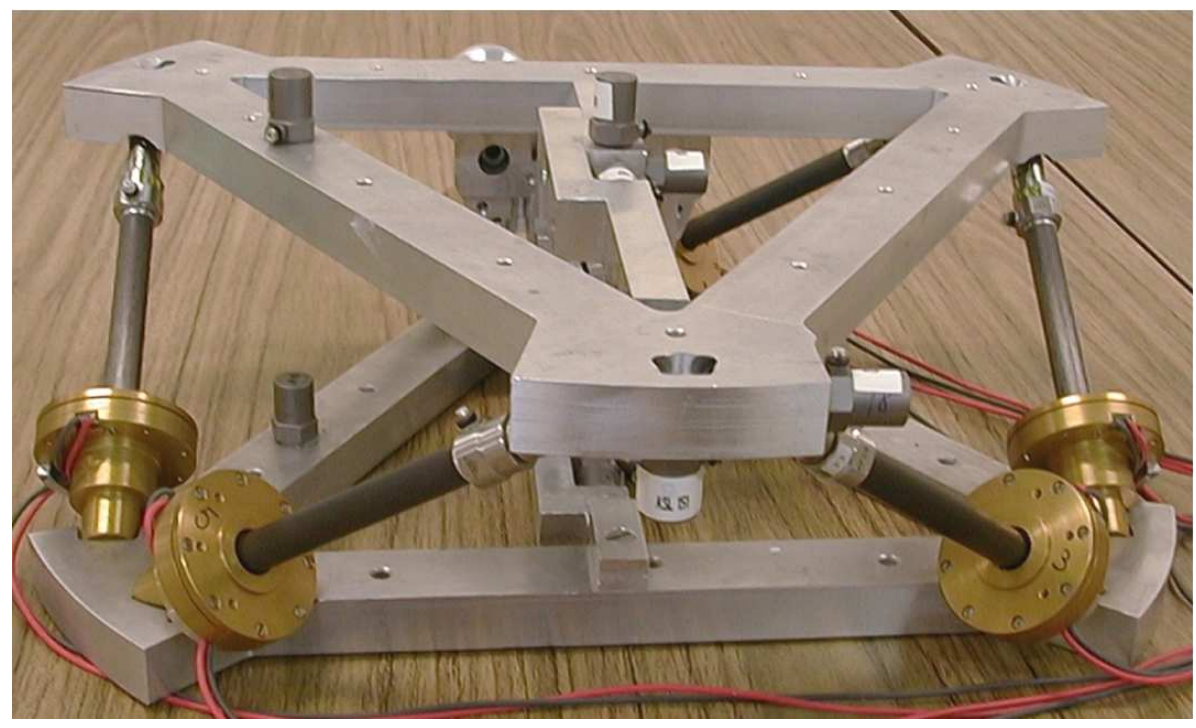

Figure 10. Six-axis active isolator based on a Stewart platform 
vides isolation between $5 \mathrm{~Hz}$ and $500 \mathrm{~Hz}$, with a maximum attenuation of $-40 \mathrm{~dB}$ near $100 \mathrm{~Hz}$. Besides the voice coil actuators and the force sensors, the system requires the conditioning electronics for the sensors and the power electronics for the actuators. Next section describes the relaxation isolator which attempts to combine a passive system (no amplifiers) with an asymptotic decay rate comparable to that of the active isolator.

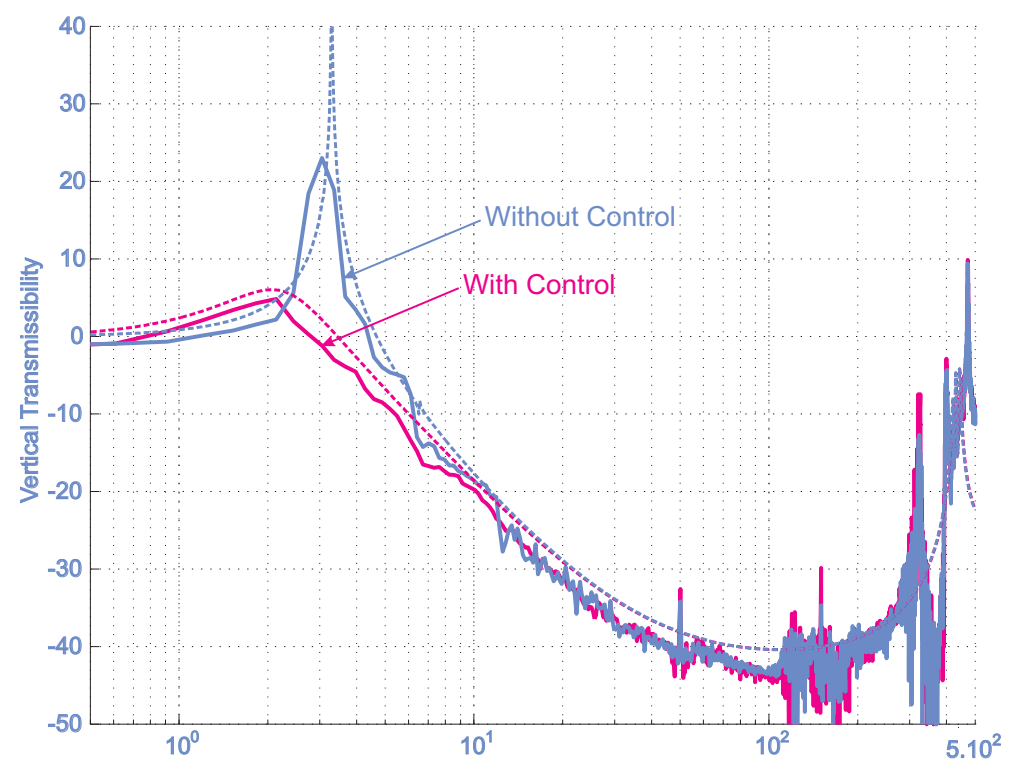

Figure 11. Vertical transmissibility of the six-axis isolator, with and without control. The full line corresponds to experimental results and the numerical simulations are in dotted line

\subsection{Relaxation isolator}

The relaxation isolator consists of a spring $k$ in parallel with a Maxwell unit $\left(k_{1}\right.$ and $c$ in series), Figure 12(a). At high frequency, this system behaves like a pure spring of stiffness $k+k_{1}$, which guarantees a asymptotic decay rate of $-40 \mathrm{~dB} /$ decade.

The transmissibility of the relaxation isolator reads:

$$
\frac{X_{1}}{X_{2}}=\frac{\left(k_{1}+c s\right) k+k_{1} c s}{\left(M s^{2}+c s+k\right)\left(k_{1}+c s\right)-c^{2} s^{2}}
$$

It is represented in Figure 13 for a fixed value of $k_{1} / k$ and various values of $c$. The high frequency behavior is as $s^{-2}$, that is $-40 \mathrm{~dB} /$ decade; the two peaks for $c=0$ and 
$c \rightarrow \infty$ correspond respectively to $\omega_{n}=\sqrt{k / m}$ and $\omega_{n} \sqrt{1+k_{1} / k}$; the minimum overshoot is obtained for:

$$
c^{o p t}=\frac{k_{1}}{\omega_{n}}\left(1+\frac{k_{1}}{k}\right)^{-3 / 4}
$$

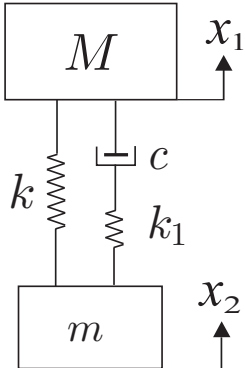

(a)

a)

$$
\begin{gathered}
c=T^{2} / R \\
k_{1}=T^{2} / L
\end{gathered}
$$

\section{$x_{2}$}

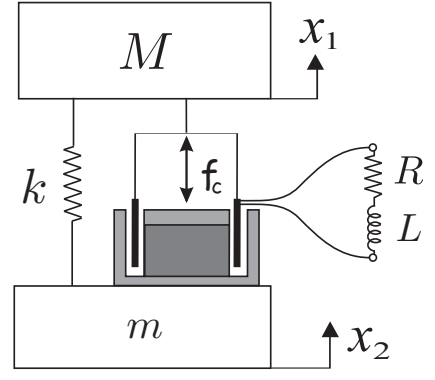

(b)

Figure 12. (a) Relaxation isolator. (b) Electromagnetic realization with a moving-coil transducer and $R-L$ circuit

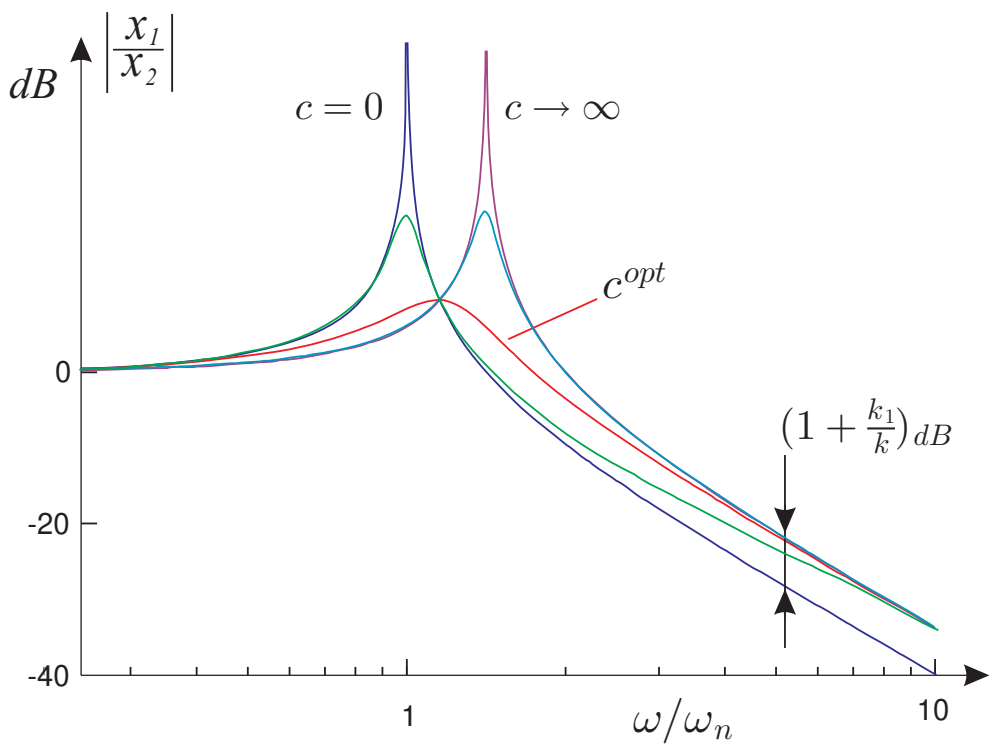

Figure 13. Transmissibility of the relaxation isolator for a fixed value of $k_{1} / k$ and various values of $c$ 


\subsection{Electromagnetic realization}

The principle of the relaxation isolator is simple but not necessarily simple to realize. In particular, the Maxwell unit can be realized with viscoelastic materials, but these are notorious for being temperature sensitive. In fact, a much more stable configuration can be achieved with an electromechanical converter (moving coil transducer) and a $R-L$ circuit, as shown in Figure 12(b). It can be shown that this system is equivalent to the previous one with (de Marneffe, 2007):

$$
k_{1}=\frac{T^{2}}{L} \quad c=\frac{T^{2}}{R}
$$

where $T$ is the moving coil transducer constant. Thus, the relaxation isolator may be adjusted by changing the parameters $T, R$ and $L$ which are likely to be much more easy to adjust and more thermally stable.

A six-axis passive isolator based on this concept and the architecture described earlier is currently in development in our laboratory on behalf of ESA; it is due to be tested in parabolic flight during 2008. Besides being electronically much simpler, the absence of a force sensor in the leg makes it possible to raise the first transverse mode to $750 \mathrm{~Hz}$, which improves the high frequency behavior. On the other hand, the overshoot at low frequency is unavoidable.

\section{Conclusion}

This paper illustrates the activity of the Active Structures Laboratory of ULB with three projects related to the dynamics and control in precision engineering. For each of them, the modelling, the control and the prototyping has been addressed.

\section{Acknowledgements}

The research on piezoelectric structures was supported by the the European FP6 Integrated Project InMAR and the European FP6 STREP program CASSEM. The research on vibration isolation was supported by ESA within the projects LSSP and PRODEX N 90147. G. Rodrigues acknowledges "Fundação para a Ciência e a Tecnologia", Portugal, (FCT) for his PhD grant SFRH/BD/21732/2005.

\section{References}

Bent A., Hagood N., “ Piezoelectric Fiber Composites with Interdigitated Electrodes”, Journal of Intelligent Material Systems and Structures, vol. 8, n¹1, p. 903-919, 1997.

de Marneffe B., Active and Passive Vibration Isolation and Damping via Shunted Transducers, $\mathrm{PhD}$ thesis, Université Libre de Bruxelles, 2007. 
Deraemaeker A., Benelechi S., Benjeddou A., Preumont A., “ Analytical and Numerical Computation of Homogenized Properties of MFCs : Applicatio $n$ to a composite boom with MFC actuators and sensors", Proc. III ECCOMAS thematic conference on Smart Structures and Materials, Gdansk, Poland, July, 2007.

Piefort V., Finite Element Modelling of Piezoelectric Active Structures, PhD thesis, Université Libre de Bruxelles, June, 2001.

Preumont A., Vibration Control of Active Structures: and Introduction, Kluwer, 2002. 2nd edition.

Preumont A., Horodinca M., Romanescu I., de Marneffe B., Avraam M., Deraemaeker A., Bossens F., Abu Hanieh A., " A Six-Axis Single-Stage Active Vibration Isolator Based on Stewart Platform”, Journal of Sound and Vibration, vol. 300, p. 644-661, 2007.

Roddier F., Adaptive Optics in Astronomy, 1st edn, Cambridge University Press, 1999.

Wilkie W., Bryant R., High J., Fox R., Helbaum R., Jalink A., Little B., Mirick P., “ Low-cost piezocomposite actuator for structural control applications", Proc. SPIE 7th Annual Int. Symp. Smart. Struct. Mater., Newport Beach, USA, 2000. 
